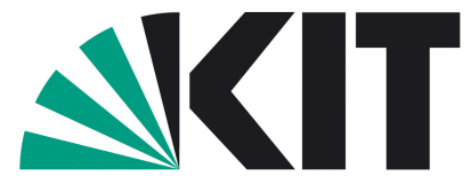

Karlsruher Institut für Technologie

\title{
On the Linearization of Operators Related to the Full Waveform Inversion in Seismology
}

\author{
A. Kirsch \\ A. Rieder \\ Preprint Nr. 13/03
}

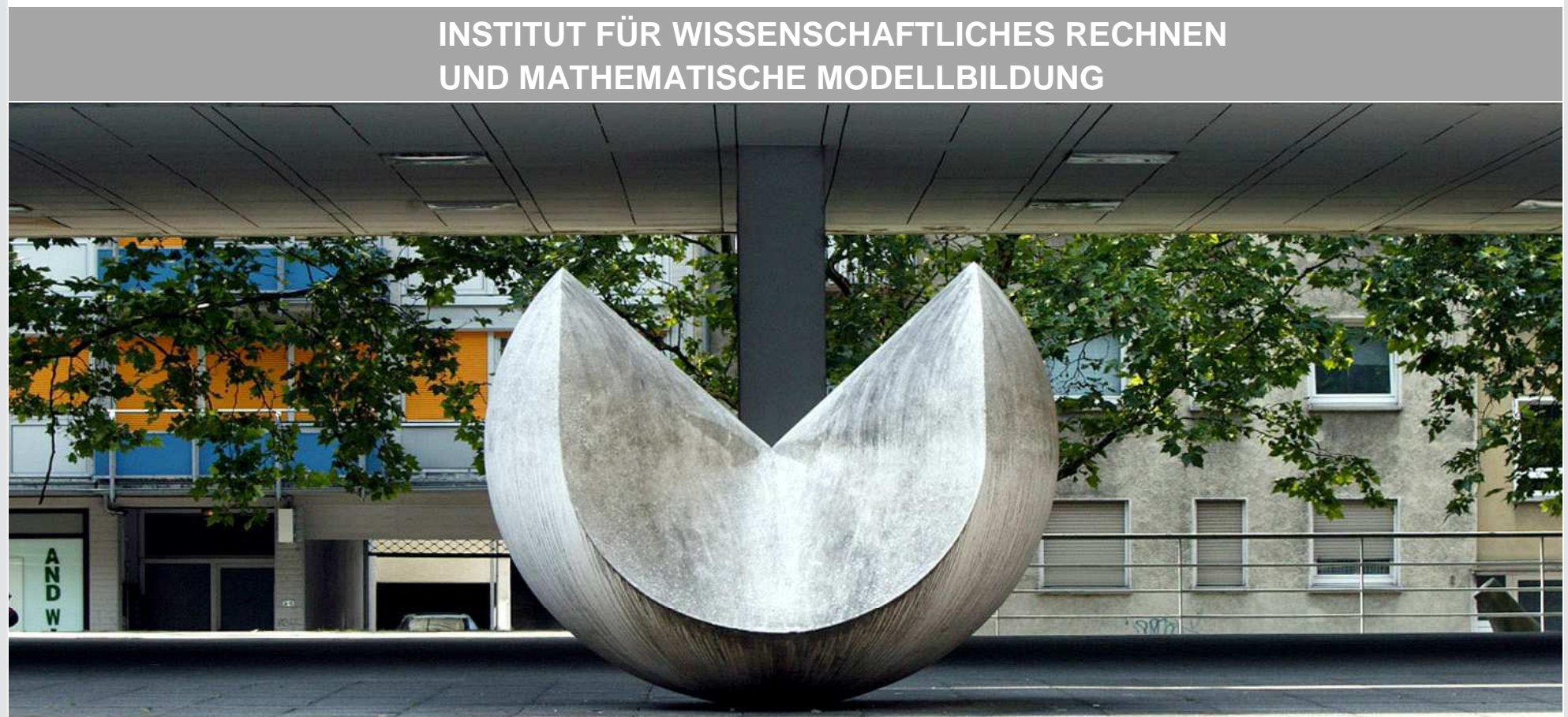

KIT - Universität des Landes Baden-Wurttemberg und

nationales Forschungszentrum in der Helmholtz-Gemeinschaft 


\section{Anschriften der Verfasser:}

Prof. Dr. Andreas Kirsch

Institut für Algebra und Geometrie

Karlsruher Institut für Technologie (KIT)

D-76128 Karlsruhe

Prof. Dr. Andreas Rieder

Institut für Angewandte und Numerische Mathematik

Karlsruher Institut für Technologie (KIT)

D-76128 Karlsruhe 


\title{
ON THE LINEARIZATION OF OPERATORS RELATED TO THE FULL WAVEFORM INVERSION IN SEISMOLOGY
}

\author{
ANDREAS KIRSCH AND ANDREAS RIEDER
}

\begin{abstract}
In this work we analyze the parameter-to-solution map of the acoustic wave equation with respect to its parameters wave speed and mass density. This map is a mathematical model for the seismic inverse problem where one wants to recover the parameters from measurements of the acoustic potential. We show its complete continuity and Fréchet differentiability. To this end we provide necessary existence, stability and regularity results. Moreover, we discuss various implications of our findings on the inverse problem and comment on the Born series.
\end{abstract}

\section{INTRODUCTION}

Our goal is to study Fréchet differentiability of the map $\mathcal{F}:(\nu, \varrho) \mapsto u$ in appropriate function spaces where $u$ solves the scalar wave equation

$$
\frac{1}{\varrho \nu^{2}} \partial_{t}^{2} u-\nabla_{\mathbf{x}} \cdot\left(\frac{1}{\varrho} \nabla_{\mathbf{x}} u\right)=f(\mathbf{x}, t),\left.\quad u\right|_{\partial \Omega}=0 .
$$

Here, $\Omega \subset \mathbb{R}^{d}$ is a bounded domain with a smooth boundary and (1) is furnished with Cauchy data

$$
u(\cdot, 0)=u_{0} ; \quad \partial_{t} u(\cdot, 0)=u_{1} .
$$

The coefficients $\nu$ and $\varrho$ are the wave speed and the mass density of the medium, respectively. They are real valued and allowed to be spatially varying: $\nu=\nu(\mathbf{x}), \varrho=\varrho(\mathbf{x})$.

The map $\mathcal{F}$ plays a prominent role in the so-called full waveform inversion in seismic imaging, see, e.g., Symes [12]. Here $\nu$ and $\varrho$ have to be recovered from the acoustic potential $\left.u\right|_{M}$ where $M$ is a certain subset of $\bar{\Omega} \times[0, \infty[$ (on $M$ the measurements are taken). Although Newton-like solvers are used in practice, sound investigations of the Fréchet differentiability of $\mathcal{F}$ under realistic assumptions are rare. In his $\mathrm{PhD}$ thesis Stolk [11] proved that $\mathcal{F}$ possesses a Gâteaux (directional) derivative which is also its Fréchet derivative as we will validate in this paper. Indeed, the solution $u^{\prime}$ of the wave equation

$$
\begin{gathered}
\frac{1}{\varrho \nu^{2}} \partial_{t}^{2} u^{\prime}-\nabla_{\mathbf{x}} \cdot\left(\frac{1}{\varrho} \nabla_{\mathbf{x}} u^{\prime}\right)=\left(\frac{2 h_{1}}{\varrho \nu^{3}}+\frac{h_{2}}{\varrho^{2} \nu^{2}}\right) \partial_{t}^{2} u-\nabla_{\mathbf{x}} \cdot\left(\frac{h_{2}}{\varrho^{2}} \nabla_{\mathbf{x}} u\right),\left.\quad u^{\prime}\right|_{\partial \Omega}=0, \\
u^{\prime}(\cdot, 0)=\partial_{t} u^{\prime}(\cdot, 0)=0,
\end{gathered}
$$

is the Fréchet derivative of $\mathcal{F}$ about $(\nu, \varrho)$ in the direction $\left(h_{1}, h_{2}\right)$ (underlying spaces and further requirements will be formulated below).

First differentiability results have been reported by Bao and Symes [2]. They studied stability properties of a formal derivative of the parameter-to-solution map of a variant of (1) (only the density determination problem was considered, however, in a half space). Bao [1] extended this line of research and showed that the formal derivative is a Fréchet derivative for densities in a certain smoothness class. Very recently - during our work on

Date: June 27, 2013. 
the present article - Blazek, Stolk, and Symes [3] came up with an elaborate consideration of first order hyperbolic systems which cover the first order formulation of the above wave equation as a special case. Thus, some of our results can be obtained from [3] under slightly stronger assumptions (we explain this in detail in the last section). Since their approach is very general and rather abstract an independent, self-contained, concrete study of the acoustic wave equation is justified, the more so as (1) is still a widely used model in seismology. Besides, we add many new aspects to this subject.

In the next section we lay the analytic foundation of this paper: We formulate the wave equation weakly, recall and provide existence and uniqueness results as well as stability (energy) estimates. Finally, we prove regularity of the acoustic potential with respect to time under regularity of the source term and the Cauchy data. Section 3 contains preparatory results to validate Fréchet differentiability of $\mathcal{F}$ which is the main topic of Section 4. We also show compactness of the Fréchet derivative and discuss its implication on the seismic inverse problem. Further, we comment on the Born series and why our results do not yield its convergence. Finally, in Section 5 we relate the work of Blazek et al. [3] to ours.

\section{The AnAlytic Setting}

In a first step we consider

$$
c \partial_{t}^{2} u-\nabla_{\mathbf{x}} \cdot\left(r \nabla_{\mathbf{x}} u\right)=f(\mathbf{x}, t),\left.\quad u\right|_{\partial \Omega}=0,
$$

with initial data (2). Let $V=H_{0}^{1}(\Omega)$ and $H=L^{2}(\Omega)$. We search for the solution $u$ in the space

$$
X:=\mathcal{C}^{0}([0, T], V) \cap \mathfrak{e}^{1}([0, T], H)
$$

equipped with the canonical norm

$$
\|u\|_{X}:=\left(\max _{0 \leq t \leq T}\|u(t)\|_{V}^{2}+\max _{0 \leq t \leq T}\|\dot{u}(t)\|_{H}^{2}\right)^{1 / 2} .
$$

The dot on top of a time dependent function indicates the time derivative. With this norm $X$ is a Banach space.

The weak formulation of (3) reads:

Given $c, r \in L^{\infty}(\Omega)$ and $u_{0} \in V$ and $u_{1} \in H$ and $f \in L^{2}([0, T] \times \Omega)=$ $L^{2}((0, T), H)$ find $u \in X$ with $u(0)=u_{0}$ and $\dot{u}(0)=u_{1}$ such that

$$
\int_{0}^{T}\left(a_{r}(u(t), v(t))-\langle c \dot{u}(t), \dot{v}(t)\rangle_{H}\right) \mathrm{d} t=\int_{0}^{T}\langle f(t), v(t)\rangle_{H} \mathrm{~d} t
$$

for all $v \in \mathcal{C}_{0}^{\infty}([0, T], V)$,

see e.g. Lions and Magenes [7, Chap. 3.8] or Stolk [11, Sec. 2.4]. Here, $\langle\cdot, \cdot\rangle_{H}$ denotes the inner product in $H$ and

$$
a_{r}: V \times V \rightarrow \mathbb{R}, \quad a_{r}(\psi, \varphi)=\int_{\Omega} r \nabla_{\mathbf{x}} \psi \cdot \nabla_{\mathbf{x}} \varphi \mathrm{d} \mathbf{x} .
$$

We assume that the coefficients $c, r \in L^{\infty}(\Omega)$ are bounded away from zero; that is,

$$
c(\mathbf{x}) \geq \mathrm{c}_{-} \text {a.e. and } r(\mathbf{x}) \geq \mathrm{r}_{-} \text {a.e. }
$$

for positive constants $c_{-}$and $r_{-}$. 
Under the above hypotheses Stolk [11, Lemma 2.4.1] has shown an energy estimate for $u$ resulting in ${ }^{1}$

$$
\|u(t)\|_{V}^{2}+\|\dot{u}(t)\|_{H}^{2} \lesssim\left\|u_{0}\right\|_{V}^{2}+\left\|u_{1}\right\|_{H}^{2}+\int_{0}^{T}\|f(\tau)\|_{H}^{2} \mathrm{~d} \tau,
$$

that is,

$$
\|u\|_{X}^{2} \lesssim\left\|u_{0}\right\|_{V}^{2}+\left\|u_{1}\right\|_{H}^{2}+\int_{0}^{T}\|f(\tau)\|_{H}^{2} \mathrm{~d} \tau .
$$

By this estimate he proved that (4) admits a unique solution $u \in X$ which depends continuously on $u_{0}, u_{1}$, and $f$, see also [7, Chap. 3.8] or the proof of Theorem 2.3 below.

Moreover, for almost all $s \in] 0, T[$ we have that

$$
a_{r}(u(s), w)+\langle c \ddot{u}(s), w\rangle_{V^{\prime} \times V}=\langle f(s), w\rangle_{H} \quad \text { for all } w \in V,
$$

where $\langle\cdot, \cdot\rangle_{V^{\prime} \times V}$ is the duality paring in $V^{\prime} \times V$. In particular

$$
c \ddot{u} \in L^{2}\left([0, T], V^{\prime}\right) \text { and } \ddot{u} \in L^{2}\left([0, T], V^{\prime}\right) \text { provided } c \in W^{1, \infty}(\Omega) .
$$

We will now verify (8) following Stolk [11, p. 23]: Let $\left\{\phi_{m}\right\}_{m \in \mathbb{N}} \subset \mathcal{C}_{0}^{\infty}(-1,1)$ be a regularizing sequence, that is, $\phi_{m} \geq 0, \int \phi_{m}(t) \mathrm{d} t=1$, and $\operatorname{supp} \phi_{m}=[-1 / m, 1 / m]$. Let $\varepsilon>0,1 / m<\varepsilon$, and $s \in[\varepsilon, T-\varepsilon]$. Then, $v(\cdot)=\phi_{m}(s-\cdot) w \in \mathcal{C}_{0}^{\infty}([0, T], V)$ for any $w \in V$. Plugged into (4) and taking into account that $\int \phi_{m}(s-t) u(t) \mathrm{d} t=\left(\phi_{m} \star u\right)(s)$ yields

$$
a_{r}\left(\left(\phi_{m} \star u\right)(s), w\right)+\left\langle c\left(\dot{\phi}_{m} \star \dot{u}\right)(s), w\right\rangle_{V^{\prime} \times V}=\left\langle\left(\phi_{m} \star f\right)(s), w\right\rangle_{H}
$$

which results in (8) for $m \rightarrow \infty$.

Example 2.1. We include an example to show that - in contrast to elliptic and parabolic boundary value problems - the weaker assumption $f \in L^{2}\left([0, T], V^{\prime}\right)$ in (4) is not sufficient to guarantee $u \in L^{2}([0, T], V)$. Let $r=1$ and $c=1$ and $\Omega$ be the square $\left.\Omega=\right] 0, \pi[\times] 0, \pi[$ in $\mathbb{R}^{2}$. The functions

$$
v_{n}(\mathbf{x})=\frac{2}{\pi|n|} \sin \left(n_{1} x_{1}\right) \sin \left(n_{2} x_{2}\right), \quad \mathbf{x}=\left(x_{1}, x_{2}\right) \in \Omega, n=\left(n_{1}, n_{2}\right) \in \mathbb{N}^{2},
$$

are eigenfunctions of $-\Delta_{\mathbf{x}}$ in $\Omega$ with respect to Dirichlet boundary conditions corresponding to the eigenvalues $\lambda_{n}=|n|^{2}$. They are normalized such that $\left\|\nabla v_{n}\right\|_{L^{2}(\Omega)}=1$ as well as $\left\|v_{n}\right\|_{L^{2}(\Omega)}=1 /|n|$. Furthermore, $\left\{v_{n}\right\}$ and $\left\{|n| v_{n}\right\}$ are complete orthonormal systems in $\left(V,\langle\nabla \cdot, \nabla \cdot\rangle_{L^{2}(\Omega)}\right)$ and $L^{2}(\Omega)$, respectively. Define the source function

$$
f(\mathbf{x}, t)=\sum_{n \in \mathbb{N}^{2}} \rho_{n}|n|^{2} \cos (|n| t) v_{n}(\mathbf{x})
$$

for any coefficients $\rho_{n}$ with $\sum_{n \in \mathbb{N}^{2}} \rho_{n}^{2}<\infty$. Then $f(t)=f(\cdot, t) \in V^{\prime}=H^{-1}(\Omega)$ as seen from the form

$$
\langle f(t), \psi\rangle_{V^{\prime} \times V}=\sum_{n, m \in \mathbb{N}^{2}} \rho_{n}|n|^{2} \cos (|n| t) \psi_{m}\left\langle v_{n}, v_{m}\right\rangle_{L^{2}(\Omega)}=\sum_{n \in \mathbb{N}^{2}} \rho_{n} \psi_{n} \cos (|n| t)
$$

\footnotetext{
${ }^{1} A \lesssim B$ indicates the existence of a generic constant $m$ such that $A \leq m B$ uniformly in all relevant parameters of the expressions $A$ and $B$. The respective context will define the meaning of 'relevant parameters'.
} 
where $\psi_{m}=\left\langle\nabla \psi, \nabla v_{m}\right\rangle_{L^{2}(\Omega)}$. The solution of $\partial_{t}^{2} u(t)-\Delta_{\mathbf{x}} u(t)=f(t)$ with $u(0)=\dot{u}(0)=0$ is given by

$$
u(t)=-\frac{1}{2} \sum_{n \in \mathbb{N}^{2}} \rho_{n}|n| t \sin (|n| t) v_{n} .
$$

Obviously, $\rho_{n}$ can be chosen such that $u(t) \in L^{2}(\Omega)$ fails to be in $V=H_{0}^{1}(\Omega)$, for instance, $\rho_{n}=1 /|n|$.

Remark 2.2. We motivate why the Dirchlet boundary restriction in (1) and (4) is - to a certain extent - physically meaningful for seismic wave propagation in free space. Indeed, due to the finite wave propagation speed homogeneous Dirichlet boundary conditions can be safely assumed in case $\Omega$ is sufficiently large: To this end consider the Cauchy problem

$$
\left.c \partial_{t}^{2} u-\nabla_{\mathbf{x}} \cdot\left(r \nabla_{\mathbf{x}} u\right)=f \quad \text { in } \mathbb{R}^{d} \times\right] 0, T[,
$$

with initial conditions $u(\cdot, 0)=u_{0}$ and $\partial_{t} u(\cdot, 0)=u_{1}$ in $\mathbb{R}^{d}$ where $f(\cdot, t), u_{0}, u_{1}$ have compact support in some ball $B(0, R)$ of radius $R$. Furthermore, $c(\mathbf{x})=c_{0}$ and $r(\mathbf{x})=1$ for all $|\mathbf{x}| \geq R$ for some constant $c_{0}>0$. Let $\Omega=B(0, \hat{R})$ be the ball of radius $\hat{R}$ which satisfies $\hat{R}>R+T / c_{0}$. For $\mathbf{z} \in \partial \Omega$ we conclude that $B\left(\mathbf{z}, T / c_{0}\right) \cap B(0, R)=\emptyset$, because $|\mathbf{x}| \geq|\mathbf{z}|-|\mathbf{x}-\mathbf{z}| \geq \hat{R}-T / c_{0}>R$ for $\mathbf{x} \in B\left(\mathbf{z}, T / c_{0}\right)$. Therefore, fixing $\mathbf{z} \in \partial \Omega$ and defining the cones

$$
C=\left\{(\mathbf{x}, t): c_{0}|\mathbf{x}-\mathbf{z}| \leq T-t\right\} \quad \text { and } \quad C_{t}=\left\{\mathbf{x}: c_{0}|\mathbf{x}-\mathbf{z}| \leq T-t\right\}
$$

we conclude that $u(\mathbf{x}, 0)$ vanishes in $C_{0}=B\left(\mathbf{z}, T / c_{0}\right)$. By a well-known result (see Evans [4]) we conclude that $u$ vanishes in all of $C$ and, in particular, $u(\mathbf{z}, t)=0$ for all $t \in[0, T]$. This holds for all $\mathbf{z} \in \partial \Omega$. Therefore, $u$ is a solution of the boundary-initial value problem (3) with $u(\cdot, 0)=u_{0}$ and $\partial_{t} u(\cdot, 0)=u_{1}$ in $\Omega$.

In our subsequent considerations we encounter a wave equation with a source term different to the one of (4):

Given $g \in \mathfrak{C}^{1}([0, T], V)$, find $w \in X$ such that $w(0)=\dot{w}(0)=0$ and

$$
\int_{0}^{T}\left(a_{r}(w(t), v(t))-\langle c \dot{w}(t), \dot{v}(t)\rangle_{H}\right) \mathrm{d} t=\int_{0}^{T} a_{r}(g(t), v(t)) \mathrm{d} t
$$

for all $v \in \mathcal{C}_{0}^{\infty}([0, T], V)$.

As we are not aware of a reference for existence, uniqueness, and stability for this setting we will provide a proof.

Theorem 2.3. Let $g \in \mathcal{C}^{1}([0, T], V)$. Then, (9) admits a unique solution $w \in X$ satisfying

$$
\|w\|_{X} \lesssim\|g\|_{\mathrm{C}^{1}([0, T], V)}
$$

where the constant in the estimate depends only on $T, \mathrm{r}_{-},\|r\|_{\infty}$, and $\mathrm{c}_{-}$.

Proof. The argument for uniqueness is as follows. Let $d$ be the difference of two solutions of (9). Then, $d$ solves (4) with data $u_{0}=u_{1}=f=0$. Thus, $d=0$ by (7).

To prove existence of a solution we generalize the approach of Example 2.1 and formulate (9) as a series of ordinary differential equations relying on the spectral theorem: There is a sequence of positive eigenvalues $\left\{\lambda_{k}\right\}_{k \in \mathbb{N}}$ converging to infinity and corresponding eigenfunctions $\left\{v_{k}\right\}_{k \in \mathbb{N}} \subset V$ such that

$$
a_{r}\left(v_{k}, \varphi\right)-\lambda_{k}\left\langle c v_{k}, \varphi\right\rangle_{H}=0 \quad \text { for all } \varphi \in V \text {. }
$$


Furthermore, the sets $\left\{v_{k}\right\}_{k \in \mathbb{N}}$ and $\left\{\sqrt{\lambda}_{k} v_{k}\right\}_{k \in \mathbb{N}}$ form complete orthonormal systems in $\left(V, a_{r}\right)$ and $\left(H,\langle c \cdot, \cdot\rangle_{H}\right)$, respectively, see, e.g., [4, Chap. 6.5]. Note that $a_{r}$ defines an inner product in $V$ which is equivalent to the ordinary inner product by Friedrich's inequality. We denote the norms in $\left(V, a_{r}\right)$ and $\left(H,\langle c \cdot, \cdot\rangle_{H}\right)$ by $\|\cdot\|_{V, a_{r}}$ and $\|\cdot\|_{H, c}$, respectively. Then we have estimates of the form

$$
\|r\|_{\infty}\|\varphi\|_{V}^{2} \geq\|\varphi\|_{V, a_{r}}^{2} \geq \mathrm{r}_{-}\|\nabla \varphi\|_{L^{2}(\Omega)}^{2} \geq \gamma^{2} \mathrm{r}_{-}\|\varphi\|_{V}^{2}, \quad \varphi \in V
$$

where $\gamma>0$ comes from Friedrich's inequality and

$$
\|c\|_{\infty}\|\psi\|_{H}^{2} \geq\|\psi\|_{H, c}^{2} \geq \mathrm{c}_{-}\|\psi\|_{H}^{2}, \quad \psi \in H
$$

We make the ansatz $w(\cdot)=\sum_{k=1}^{\infty} \omega_{k}(\cdot) v_{k}$ where the $\omega_{k}$ 's are given as unique solutions of the initial value problems

$$
\ddot{\omega}_{k}(t)+\lambda_{k}^{2} \omega_{k}(t)=\lambda_{k}^{2} g_{k}(t), \quad \omega_{k}(0)=\dot{\omega}_{k}(0)=0,
$$

where $g_{k}(t)=a_{r}\left(g(t), v_{k}\right)$. Note that $\sum_{k=1}^{\infty} g_{k}(t) v_{k}=g(t)$ and $\|g(t)\|_{V, a_{r}}^{2}=\sum_{k=1}^{\infty}\left|g_{k}(t)\right|^{2}$ and $\|\dot{g}(t)\|_{V, a_{r}}^{2}=\sum_{k=1}^{\infty}\left|\dot{g}_{k}(t)\right|^{2}$.

Assume for the moment that we have shown already that $w \in X=\mathcal{C}^{0}([0, T], V) \cap$ $\mathrm{C}^{1}([0, T], H)$. Then, $w$ solves $(9)$. Indeed, $w(0)=\dot{w}(0)=0$ and for any $\varphi \in \mathfrak{C}_{0}^{\infty}([0, T], V)$ with $\varphi(t)=\sum_{\ell} \varphi_{\ell}(t) v_{\ell}$, we have

$$
\begin{aligned}
\int_{0}^{T} & \left(a_{r}(w(t), \varphi(t))-\langle c \dot{w}(t), \dot{\varphi}(t)\rangle_{H}\right) \mathrm{d} t \\
& =\sum_{k, \ell} \int_{0}^{T}\left(\omega_{k}(t) \varphi_{\ell}(t) a_{r}\left(v_{k}, v_{\ell}\right)-\dot{\omega}_{k}(t) \dot{\varphi}_{\ell}(t)\left\langle c v_{k}, v_{\ell}\right\rangle_{H}\right) \mathrm{d} t \\
& =\sum_{k} \int_{0}^{T}\left(\omega_{k}(t) \varphi_{k}(t)-\frac{1}{\lambda_{k}^{2}} \dot{\omega}_{k}(t) \dot{\varphi}_{k}(t)\right) \mathrm{d} t=\sum_{k} \int_{0}^{T}\left(\omega_{k}(t)+\frac{1}{\lambda_{k}^{2}} \ddot{\omega}_{k}(t)\right) \varphi_{k} \mathrm{~d} t \\
& =\sum_{k} \int_{0}^{T} g_{k}(t) \varphi_{k}(t) \mathrm{d} t=\int_{0}^{T} a_{r}(g(t), \varphi(t)) \mathrm{d} t .
\end{aligned}
$$

Finally, we verify the stability estimate which shows that $w \in X=\mathcal{C}^{0}([0, T], V) \cap$ $\mathrm{C}^{1}([0, T], H)$. We note that $\omega_{k}$ is explicitly given by

$$
\omega_{k}(t)=\lambda_{k} \int_{0}^{t} \sin \left(\lambda_{k}(t-s)\right) g_{k}(s) \mathrm{d} s, \quad t \in[0, T] .
$$

By partial integration we rewrite this as

$$
\omega_{k}(t)=g_{k}(t)-\cos \left(\lambda_{k} t\right) g_{k}(0)-\int_{0}^{t} \cos \left(\lambda_{k}(t-s)\right) \dot{g}_{k}(s) \mathrm{d} s, \quad t \in[0, T] .
$$

From this we observe that

$$
\left\|\omega_{k}\right\|_{\infty} \leq 2\left\|g_{k}\right\|_{\infty}+\sqrt{T}\left\|\dot{g}_{k}\right\|_{L^{2}(0, T)} \lesssim\left\|g_{k}\right\|_{L^{2}(0, T)}+\left\|\dot{g}_{k}\right\|_{L^{2}(0, T)}
$$

where the constant depends on $T$ only. Analogously,

$$
\begin{aligned}
\dot{\omega}_{k}(t) & =\lambda_{k}^{2} \int_{0}^{t} \cos \left(\lambda_{k}(t-s)\right) g_{k}(s) \mathrm{d} s \\
& =\lambda_{k} \sin \left(\lambda_{k} t\right) g_{k}(0)+\lambda_{k} \int_{0}^{t} \sin \left(\lambda_{k}(t-s)\right) \dot{g}_{k}(s) \mathrm{d} s, \quad t \in[0, T],
\end{aligned}
$$


and thus

$$
\left\|\dot{\omega}_{k}\right\|_{\infty} \leq \lambda_{k}\left\|g_{k}\right\|_{\infty}+\lambda_{k} \sqrt{T}\left\|\dot{g}_{k}\right\|_{L^{2}(0, T)} \lesssim \lambda_{k}\left(\left\|g_{k}\right\|_{L^{2}(0, T)}+\left\|\dot{g}_{k}\right\|_{L^{2}(0, T)}\right) .
$$

Again, the constant depends on $T$ only. Defining the partial sums $w^{n}(t)=\sum_{k=1}^{n} \omega_{k}(t) v_{k}$ we note that for $m>n$

$$
\begin{aligned}
\max _{0 \leq t \leq T}\left\|w^{n}(t)-w^{m}(t)\right\|_{V, a_{r}}^{2} & =\max _{0 \leq t \leq T} \sum_{k=n+1}^{m} \omega_{k}(t)^{2} \leq \sum_{k=n+1}^{m}\left\|\omega_{k}\right\|_{\infty}^{2} \\
& \lesssim \sum_{k=n+1}^{m}\left(\left\|g_{k}\right\|_{L^{2}(0, T)}^{2}+\left\|\dot{g}_{k}\right\|_{L^{2}(0, T)}^{2}\right) .
\end{aligned}
$$

Analogously, we make use of the fact that $\left\{\lambda_{k} v_{k}: k \in \mathbb{N}\right\}$ is an orthonormal system in $\left(H,\langle c \cdot, \cdot\rangle_{H}\right)$. Therefore,

$$
\begin{aligned}
\max _{0 \leq t \leq T}\left\|\dot{w}^{n}(t)-\dot{w}^{m}(t)\right\|_{H, c}^{2} & =\max _{0 \leq t \leq T} \sum_{k=n+1}^{m} \frac{1}{\lambda_{k}^{2}} \dot{\omega}_{k}(t)^{2} \leq \sum_{k=n+1}^{m} \frac{1}{\lambda_{k}^{2}}\left\|\dot{\omega}_{k}\right\|_{\infty}^{2} \\
& \lesssim \sum_{k=n+1}^{m}\left(\left\|g_{k}\right\|_{L^{2}(0, T)}^{2}+\left\|\dot{g}_{k}\right\|_{L^{2}(0, T)}^{2}\right) .
\end{aligned}
$$

Again, the constants in the estimates depend on $T$ only. Combining these estimates yields with (11) and (12)

$$
\begin{aligned}
\left\|w^{n}-w^{m}\right\|_{X}^{2} & \leq \frac{1}{\gamma \sqrt{\mathrm{r}_{-}}} \max _{0 \leq t \leq T}\left\|w^{n}(t)-w^{m}(t)\right\|_{V, a_{r}}+\frac{1}{\sqrt{\mathrm{c}_{-}}} \max _{0 \leq t \leq T}\left\|\dot{w}^{n}(t)-\dot{w}^{m}(t)\right\|_{H, c} \\
& \lesssim \sum_{k=n+1}^{m}\left(\left\|g_{k}\right\|_{L^{2}(0, T)}^{2}+\left\|\dot{g}_{k}\right\|_{L^{2}(0, T)}^{2}\right)
\end{aligned}
$$

where the constant in the estimate depends on $T, \mathrm{r}_{-}$, and $\mathrm{c}_{-}$only. This shows that $\left\{w^{n}\right\}$ is a Cauchy sequence in $X$ because of the convergence

$$
\begin{aligned}
\sum_{k=1}^{\infty}\left(\left\|g_{k}\right\|_{L^{2}(0, T)}^{2}+\left\|\dot{g}_{k}\right\|_{L^{2}(0, T)}^{2}\right) & =\int_{0}^{T}\left(\|g(t)\|_{V, a_{r}}^{2}+\|\dot{g}(t)\|_{V, a_{r}}^{2}\right) \mathrm{d} t \\
& \leq\|r\|_{\infty}\left[\|g\|_{L^{2}((0, T), V)}^{2}+\|\dot{g}\|_{L^{2}((0, T), V)}^{2}\right] .
\end{aligned}
$$

Therefore, $w \in X$, and the proof is complete.

In the remainder of this section we study how higher regularity of $u_{0}, u_{1}$, and $f$ transfers to the solution of (4). Similar results can be found, e.g., in [13, §30] or [4, Sec. 7.2.3]. For the sake of completeness and self-containedness we present elementary proofs.

Recall that $a_{r}, r \in L^{\infty}(\Omega)$, induces a bounded operator $A_{r}: V \rightarrow V^{\prime}$ by $a_{r}(v, w)=$ $\left\langle A_{r} v, w\right\rangle_{V^{\prime} \times V}=\left\langle A_{r} w, v\right\rangle_{V^{\prime} \times V}$ for all $v, w \in V$, that is, $A_{r} v=-\nabla_{\mathbf{x}} \cdot\left(r \nabla_{\mathbf{x}} v\right)$.

Lemma 2.4. Let $f \in \mathcal{C}^{1}([0, T], H)$. Further, let $u_{1}$ be in $V$ and let $u_{0} \in V$ satisfy $A_{r} u_{0} \in H$. Then,

$$
u \in \mathcal{C}^{1}([0, T), V) \cap \mathcal{C}^{2}([0, T], H) \quad \text { and } \quad c \dddot{u} \in L^{2}\left([0, T], V^{\prime}\right) .
$$

In particular, $\dot{u} \in X$. Furthermore,

$$
\|u\|_{X}+\|\dot{u}\|_{X} \lesssim\left\|u_{1}\right\|_{V}+\left\|A_{r} u_{0}\right\|_{H}+\|f\|_{\mathcal{C}^{1}([0, T], V)}
$$

where the constant in the estimate depends only on $T, \mathrm{r}_{-},\|r\|_{\infty}, \mathrm{c}_{-}$, and $\|c\|_{\infty}$. 
Proof. We formulate (8) as

$$
\left.c \ddot{u}(t)=f(t)-A_{r} u(t), \quad t \in\right] 0, T[,
$$

where the equality holds in $V^{\prime}$. Taking the limit $t \rightarrow 0$ shows that $c \ddot{u}(0)=f(0)-A_{r} u_{0} \in H$ due to our assumptions on $f$ and $u_{0}$ and thus also $\ddot{u}(0) \in H$. Therefore, the following problem is well defined with a unique solution $w \in X$ :

Find $w \in X$ with $w(0)=u_{1} \in V$ and $\dot{w}(0)=\ddot{u}(0) \in H$ such that

$$
\int_{0}^{T}\left(a_{r}(w(t), v(t))-\langle c \dot{w}(t), \dot{v}(t)\rangle_{H}\right) \mathrm{d} t=\int_{0}^{T}\langle\dot{f}(t), v(t)\rangle_{H} \mathrm{~d} t \quad \text { for all } v \in \mathcal{C}_{0}^{\infty}([0, T], V) .
$$

We are done if we can show that $\dot{u}(t) \in V$. Indeed, then $\dot{u}$ solves above problem with the correct initial values which yields $w=\dot{u}$. Also the estimate (14) holds by (7). ${ }^{2}$

Let $\eta \in \mathbb{R}$ such that $0<|\eta|<T$. Define $t_{\min }=t_{\min }(\eta)=\max \{0,-\eta\}$ and $t_{\max }=$ $t_{\max }(\eta)=\min \{T, T-\eta\}$. By construction $0 \leq t_{\min }<t_{\max } \leq T$. Further, let $D_{\eta} \ell(t)=$ $(\ell(t+\eta)-\ell(t)) / \eta$ for any time dependent function $\ell$. Consider now the problem:

Find $w_{\eta} \in \mathfrak{C}^{0}\left(\left[t_{\min }, t_{\max }\right], V\right) \cap \mathcal{C}^{1}\left(\left[t_{\min }, t_{\max }\right], H\right)$ with $w_{\eta}\left(t_{\min }\right)=D_{\eta} u\left(t_{\min }\right) \in$ $V$ and $\dot{w}_{\eta}\left(t_{\text {min }}\right)=D_{\eta} \dot{u}\left(t_{\text {min }}\right) \in H$ such that

$$
\int_{t_{\min }}^{t_{\max }}\left(a_{r}\left(w_{\eta}(t), v_{\eta}(t)\right)-\left\langle c \dot{w}_{\eta}(t), \dot{v}_{\eta}(t)\right\rangle_{H}\right) \mathrm{d} t=\int_{t_{\min }}^{t_{\max }}\left\langle D_{\eta} f(t), v_{\eta}(t)\right\rangle_{H} \mathrm{~d} t
$$

for all $v_{\eta} \in \mathcal{C}_{0}^{\infty}\left(\left[t_{\min }, t_{\max }\right], V\right)$.

Since $\mathcal{C}_{0}^{\infty}\left(\left[t_{\min }, t_{\max }\right], V\right) \subset \mathcal{C}_{0}^{\infty}([0, T], V)$ we have $w_{\eta}(t)=D_{\eta} u(t), t \in\left[t_{\min }, t_{\max }\right]$, which can be verified by the following line of arguments: subtract (8) for $s=t$ from (8) for $s=$ $t+\eta$, divide the difference by $\eta$, set $w=v_{\eta}(t)$ and finally integrate over $\left[t_{\min }, t_{\max }\right]$. Thus, $D_{\eta} u$ satisfies (16) with the right initial values and the corresponding energy estimate (6) reads

$$
\left\|D_{\eta} u(t)\right\|_{V}^{2}+\left\|D_{\eta} \dot{u}(t)\right\|_{H}^{2} \lesssim\left\|D_{\eta} u\left(t_{\min }\right)\right\|_{V}^{2}+\left\|D_{\eta} \dot{u}\left(t_{\min }\right)\right\|_{H}^{2}+\int_{t_{\min }}^{t_{\max }}\left\|D_{\eta} f(\tau)\right\|_{H}^{2} \mathrm{~d} \tau
$$

where $t \in] t_{\min }, t_{\max }[$. Letting $\eta$ approach 0 results in $\dot{u}(t) \in V$ and the proof is complete.

By an inductive argument we get even higher regularity.

Corollary 2.5. Let $f \in \mathrm{C}^{k}([0, T], H), k \geq 2$, and assume that $u_{0}, u_{1} \in V$. Define

$$
u_{i}:=c^{-1}\left(f^{(i-2)}(0)-A_{r} u_{i-2}\right), i=2, \ldots, k+1,
$$

and assume that $u_{i} \in V$ for $i=2, \ldots, k$ and $A_{r} u_{k-1} \in H$. Then $u_{i}=u^{(i)}(0)$ for all $i=0, \ldots, k+1$ and

$$
u \in \mathfrak{e}^{k}([0, T], V) \cap \mathrm{e}^{k+1}([0, T], H) .
$$

Proof. We will only sketch the arguments. Assume the result to hold true for $k(k=1$ is assured by Lemma 2.4 above), that is, $u^{(k)} \in X$ uniquely satisfies $u^{(k)}(0)=u_{k}$ and $u^{(k+1)}(0)=u_{k+1}$ and

$$
\int_{0}^{T}\left(a_{r}\left(u^{(k)}(t), v(t)\right)-\left\langle c u^{(k+1)}(t), \dot{v}(t)\right\rangle_{H}\right) \mathrm{d} t=\int_{0}^{T}\left\langle f^{(k)}(t), v(t)\right\rangle_{H} \mathrm{~d} t
$$

for all $v \in \mathcal{C}_{0}^{\infty}([0, T], V)$. Now, we are in a position to carry over the proof of Lemma 2.4.

\footnotetext{
${ }^{2}$ Note that the constant in $(7)$ depends only on $T, \mathrm{r}_{-},\|r\|_{\infty}, \mathrm{c}_{-}$, and $\|c\|_{\infty}$.
} 


\section{The Parameter To Solution Operator And Its Properties}

Now we are able to define and study the forward map

$$
F: \mathrm{D}(F) \subset L^{\infty}(\Omega) \times L^{\infty}(\Omega) \rightarrow X, \quad(c, r) \mapsto u,
$$

where $u \in X$ solves (3) in the weak sense (4) and the domain of definition of $F$ is

$$
\mathrm{D}(F)=\left\{(c, r) \in L^{\infty}(\Omega) \times L^{\infty}(\Omega): c(\mathbf{x}) \geq \mathrm{k}_{-}, r(\mathbf{x}) \geq \mathrm{k}_{-}, \text {a.e. }\right\}
$$

where $\mathrm{k}_{-}:=\max \left\{\mathrm{c}_{-}, \mathrm{r}_{-}\right\}$. First we show a compactness result.

Lemma 3.1. Let $f \in \mathfrak{C}^{1}([0, T], H), u_{0}=0$ and $u_{1} \in V$. Then, $F$ is uniformly Lipschitz continuous on bounded subsets of $\mathrm{D}(F)$.

Proof. Let $\left(r_{j}, c_{j}\right) \in \mathrm{D}(F), j=1,2$, and set $u_{j}=F\left(r_{j}, c_{j}\right)$. By Lemma 2.4 we note that $u_{j} \in \tilde{X}=\mathcal{C}^{1}([0, T], V) \cap \mathcal{C}^{2}([0, T], H)$. We substract the variational equations for $u_{1}$ and $u_{2}$ and have for $\psi \in X$ with $\psi(0)=\psi(T)=0$ :

$$
\begin{aligned}
\int_{0}^{T} & {\left[\left\langle\dot{u}_{1}(t)-\dot{u}_{2}(t), c_{2} \dot{\psi}(t)\right\rangle_{H}-a_{r_{2}}\left(u_{1}(t)-u_{2}(t)\right)\right] \mathrm{d} t } \\
& =\int_{0}^{T}\left\langle\left(c_{2}-c_{1}\right) \dot{u}_{1}(t), \dot{\psi}(t)\right\rangle_{H} d t-\int_{0}^{T} a_{r_{2}-r_{1}}\left(u_{1}(t), \psi(t)\right) \mathrm{d} t \\
& =\int_{0}^{T}\left\langle\left(c_{1}-c_{2}\right) \ddot{u}_{1}(t), \psi(t)\right\rangle_{H} \mathrm{~d} t-\int_{0}^{T} a_{r_{2}-r_{1}}\left(u_{1}(t), \psi(t)\right) \mathrm{d} t .
\end{aligned}
$$

Now, for every $t \in[0, T]$ we determine $w(t) \in V$ with

$$
a_{r_{2}}(w, \psi)=a_{r_{2}-r_{1}}\left(u_{1}, \psi\right) \text { for all } \psi \in V .
$$

The differentiability properties of $u_{1}$ yield that also $w \in \mathcal{C}^{1}([0, T], V)$ and

$$
\|w\|_{\mathcal{C}^{1}([0, T], V)} \leq \mathrm{r}_{-}^{-1}\left\|r_{2}-r_{1}\right\|_{\infty}\left\|u_{1}\right\|_{\mathcal{C}^{1}([0, T], V)} .
$$

Thus,

$$
\begin{aligned}
\int_{0}^{T}\left[\left\langle\dot{u}_{1}(t)-\dot{u}_{2}(t), c_{2} \dot{\psi}(t)\right\rangle_{H}\right. & \left.-a_{r_{2}}\left(u_{1}(t)-u_{2}(t), \psi(t)\right)\right] \mathrm{d} t \\
& =\int_{0}^{T}\left\langle\left(c_{1}-c_{2}\right) \ddot{u}_{1}(t), \psi(t)\right\rangle_{H} \mathrm{~d} t-\int_{0}^{T} a_{r_{2}}(w(t), \psi(t)) \mathrm{d} t
\end{aligned}
$$

for $\psi \in X$ with $\psi(0)=\psi(T)=0$. Therefore, the difference $u_{1}-u_{2}$ satisfies the inhomogeneous wave equation with a source term being the sum of the source terms of (4) and (9) where $f(t)=\left(c_{1}-c_{2}\right) \ddot{u}_{1}(t)$ and $g=w$, respectively. Furthermore, $u_{1}-u_{2}$ satisfies homogeneous initial conditions. Formulas (7) and (10) yield

$$
\begin{aligned}
\left\|u_{1}-u_{2}\right\|_{X} & \lesssim\left\|c_{1}-c_{2}\right\|_{\infty}\left\|\ddot{u}_{1}\right\|_{L^{2}((0, T) \times \Omega)}+\|w\|_{\mathrm{e}^{1}([0, T], V)} \\
& \lesssim\left\|c_{1}-c_{2}\right\|_{\infty}+\left\|r_{1}-r_{2}\right\|_{\infty}
\end{aligned}
$$

which proves the assertion since the constant is uniformly bounded on bounded subsets of $\mathrm{D}(F)$.

Theorem 3.2. Let $f \in \mathcal{C}^{1}([0, T], H), u_{0}=0$ and $u_{1} \in V$. The map $F:(c, r) \mapsto u$ is completely continuous from $\mathrm{D}(F)$ into $\mathrm{C}([0, T], H)$, that is, it is continuous and maps bounded sets of $\mathrm{D}(F)$ into relative compact sets in $\mathrm{C}([0, T], H)$. Here, $u \in X$ solves (3) in the weak sense (4). 
Proof. First we show that $F$ maps bounded sets of $\mathrm{D}(F)$ into bounded sets of $\tilde{X}=$ $\mathrm{C}^{1}([0, T], V) \cap \mathrm{C}^{2}([0, T], H)$. Let $Q \subset \mathrm{D}(F)$ be bounded, that is, there exist $\mathrm{k}_{+}>0$ with $\mathrm{k}_{-} \leq c(\mathbf{x}) \leq \mathrm{k}_{+}$and $\mathrm{k}_{-} \leq r(\mathbf{x}) \leq \mathrm{k}_{+}$for almost all $\mathbf{x} \in \Omega$. Let $F(c, r)=u \in \tilde{X}$ be the corresponding solution. Note that $u \in \tilde{X}$ by Lemma 2.4. Formula (14) yields

$$
\|u\|_{X}+\|\dot{u}\|_{X} \lesssim\left\|u_{1}\right\|_{V}+\|f\|_{\mathrm{e}^{1}([0, T], V)}
$$

where the constant in the estimate depends only on $T, \mathrm{k}_{-}$, and $\mathrm{k}_{+}$. This shows boundedness of $F(Q)$ in $\tilde{X}$. We show now that $F(Q)$ is relatively compact in $\mathcal{C}([0, T], H)$ by the (general) theorem of Arcela-Ascoli, see, e.g., [6, Theorem 3.1]. Indeed, for any $t \in[0, T]$ we have that $\{u(t): u \in F(Q)\} \subset\left\{v \in V:\left\|\nabla_{\mathbf{x}} v\right\|_{L^{2}(\Omega)^{d}} \leq \hat{c}\right\}$ for some $\hat{c}$, and the latter set is relatively compact in $H=L^{2}(\Omega)$. Furthermore, $F(Q)$ is equi-continuous because for $u \in F(Q)$

$$
\begin{aligned}
\left\|u\left(t_{2}\right)-u\left(t_{1}\right)\right\|_{H} & =\sup _{\|\psi\|_{H}=1}\left\langle u\left(t_{2}\right)-u\left(t_{1}\right), \psi\right\rangle_{H}=\sup _{\|\psi\|_{H}=1} \int_{t_{1}}^{t_{2}} \frac{\mathrm{d}}{\mathrm{d} s}\langle u(s), \psi\rangle_{H} \mathrm{~d} s \\
& =\sup _{\|\psi\|_{H}=1} \int_{t_{1}}^{t_{2}}\langle\dot{u}(s), \psi\rangle_{H} \mathrm{~d} s \leq\left|t_{2}-t_{1}\right|\|\dot{u}\|_{\mathcal{e}([0, T], H)} \leq \tilde{c}\left|t_{2}-t_{1}\right| .
\end{aligned}
$$

The continuity of $F$ is due to Lemma 3.1.

3.1. Partial derivative with respect to $c$. Under regularity assumptions on the data $u_{0}, u_{1}$, and $f$ we can show Fréchet differentiability of $F$ as defined in (17).

Theorem 3.3. In (4) let $u_{0}=0, u_{1} \in V$ with $A_{r} u_{1} \in H$, and $f \in \mathcal{C}^{2}([0, T], H)$ with $f(0)=0$. Then, at every $(c, r) \in \mathrm{D}(F)$ the Fréchet derivative of $F(\cdot, r)$ with respect to $c$ is the bounded linear operator $\partial_{1} F(c, r) \in \mathcal{L}\left(L^{\infty}(\Omega), X\right)$ defined by $\partial_{1} F(c, r) h=u^{\prime}$ where $u^{\prime} \in X$ uniquely solves

$$
\int_{0}^{T}\left(a_{r}\left(u^{\prime}(t), v(t)\right)-\left\langle c \dot{u}^{\prime}(t), \dot{v}(t)\right\rangle_{H}\right) \mathrm{d} t=\int_{0}^{T}\langle h \dot{u}(t), \dot{v}(t)\rangle_{H} \mathrm{~d} t
$$

for all $v \in \mathcal{C}_{0}^{\infty}([0, T], V)$ with initial data $u^{\prime}(0)=0, \dot{u}^{\prime}(0)=0$, where $u$ is the solution of (4).

Proof. The hypotheses of Corollary 2.5 are met for $k=2$. Hence, $\ddot{u}(t) \in H$ (even in $V$ ) and the right hand side of (18) can be formulated as

$$
\int_{0}^{T}\langle h \dot{u}(t), \dot{v}(t)\rangle_{H} \mathrm{~d} t=-\int_{0}^{T}\langle h \ddot{u}(t), v(t)\rangle_{H} \mathrm{~d} t .
$$

From (7) we get

$$
\left\|u^{\prime}\right\|_{X}^{2} \lesssim\|h\|_{\infty}^{2} \int_{0}^{T}\|\ddot{u}(\tau)\|_{H}^{2} \mathrm{~d} \tau
$$

Recall that $\dot{u}$ satisfies (4) with $f$ replaced by $\dot{f}$ and Cauchy data $\dot{u}(0)=u_{1}$ and $\ddot{u}(0)=$ $c^{-1}\left(f(0)-A_{r} u_{0}\right)=0$, that is, an energy estimate like (6) holds and yields

$$
\|\ddot{u}(\tau)\|_{H}^{2} \lesssim\left\|u_{1}\right\|_{V}^{2}+\int_{0}^{T}\|\dot{f}(s)\|_{H}^{2} \mathrm{~d} s \quad \text { for all } \tau .
$$

Hence, $\partial_{1} F(c, r) \in \mathcal{L}\left(L^{\infty}(\Omega, X)\right)$. Up to here we only made use of the regularity assumptions $k=1$ of Corollary 2.5.

Next we verify that $\partial_{1} F(c, r)$ is the Fréchet derivative of $F$ at $c$. Let $h \in L^{\infty}(\Omega)$ be such that $c+h \geq \mathrm{k}_{-}$a.e. and denote by $u^{+}$the solution of (4) where $c$ is replaced by 
$c+h$. Note that the data $u_{0}, u_{1}$, and $f$ are such that $u^{+}$has the same regularity as $u$ (Corollary 2.5). Then, $d:=u^{+}-u-u^{\prime} \in X$ satisfies $d(0)=\dot{d}(0)=0$ as well as

$$
\begin{gathered}
\int_{0}^{T}\left(a_{r}(d(t), v(t))-\langle c \dot{d}(t), \dot{v}(t)\rangle_{H}\right) \mathrm{d} t=\int_{0}^{T}\left\langle h\left(\dot{u}^{+}(t)-\dot{u}(t)\right), \dot{v}(t)\right\rangle_{H} \mathrm{~d} t \\
=-\int_{0}^{T}\left\langle h\left(\ddot{u}^{+}(t)-\ddot{u}(t)\right), v(t)\right\rangle_{H} \mathrm{~d} t \quad \text { for all } v \in \mathcal{C}_{0}^{\infty}([0, T], V) .
\end{gathered}
$$

From (7) we obtain

$$
\|d\|_{X}^{2} \lesssim\|h\|_{\infty}^{2} \int_{0}^{T}\|\ddot{\delta}(\tau)\|_{H}^{2} \mathrm{~d} \tau \quad \text { where } \delta(t)=u^{+}(t)-u(t) .
$$

By (8),

(19) $\quad a_{r}(\delta(s), w)+\langle c \ddot{\delta}(s), w\rangle_{V^{\prime} \times V}=-\left\langle h \ddot{u}^{+}(s), w\right\rangle_{H} \quad$ for all $w \in V$ and almost all $s$.

Differentiating (19) and subsequent integration we see that $\dot{\delta} \in X$ is the unique solution of

$\int_{0}^{T}\left(a_{r}(\dot{\delta}(t), v(t))-\langle c \ddot{\delta}(t), \dot{v}(t)\rangle_{H}\right) \mathrm{d} t=-\int_{0}^{T}\left\langle h \dddot{u}^{+}(t), v(t)\right\rangle_{H} \mathrm{~d} t$ for all $v \in \mathcal{C}_{0}^{\infty}([0, T], V)$

with Cauchy data $\dot{\delta}(0))=\ddot{\delta}(0)=0$. An application of (6)

$$
\|\ddot{\delta}(t)\|_{H}^{2} \lesssim\|h\|_{\infty}^{2} \int_{0}^{T}\left\|\dddot{u}^{+}(\tau)\right\|_{H}^{2} \mathrm{~d} \tau \lesssim\|h\|_{\infty}^{2} \quad \text { as } h \rightarrow 0
$$

ends the proof.

The assumptions $f(0)=0$ and $u_{0}=0$ on the data in Theorem 3.3 have been necessary to guarantee that both $u=F(c, r)$ and $u^{+}=F(c+h, r)$ have the same regularity for all $h \in L^{\infty}(\Omega)$ with $c+h \geq \mathrm{k}_{-}$:

$$
(c+h)^{-1}\left(f(0)-A_{r} u_{0}\right)=0 \in V .
$$

If we allow higher regularity of $c$ then we can weaken these assumptions.

Corollary 3.4. Let $u_{0} \in V, u_{1} \in V$, and $f \in \mathfrak{C}^{2}([0, T], H)$ satisfy $f(0)-A_{r} u_{0} \in V$ and $A_{r} u_{1} \in H$. Define

$$
\widetilde{F}: \mathrm{D}(\widetilde{F}) \subset W^{1, \infty}(\Omega) \times L^{\infty}(\Omega) \rightarrow X, \quad(c, r) \mapsto u,
$$

where $u$ solves (4) and

$$
\mathrm{D}(\widetilde{F})=\left\{(\lambda, \kappa) \in W^{1, \infty}(\Omega) \times L^{\infty}(\Omega): \lambda(\mathbf{x}) \geq \mathrm{k}_{-}, \kappa(\mathbf{x}) \geq \mathrm{k}_{-} \text {a.e. }\right\} .
$$

Then, $\widetilde{F}$ is Fréchet differentiable with respect to $c$ and $\partial_{1} \widetilde{F}(c, r) \in \mathcal{L}\left(W^{1, \infty}(\Omega), X\right)$ where $\partial_{1} \widetilde{F}(c, r) h=u^{\prime}$ with $u^{\prime}$ from (18).

Proof. Both, $u$ and $u^{+}$have the regularity to carry over the above proof to the present situation. 


\subsection{Partial derivative with respect to $r$.}

Theorem 3.5. In (4) let $u_{0}=0, u_{1} \in V$, and let $f \in \mathcal{C}^{1}([0, T], H)$. Then, for every $(c, r) \in \mathrm{D}(F)$ the Fréchet derivative of $F(c, \cdot)$ with respect to $r$ exists and is the bounded linear operator $\partial_{2} F(c, r) \in \mathcal{L}\left(L^{\infty}(\Omega), X\right)$ defined by $\partial_{2} F(c, r) h=u^{\prime}$ where $u^{\prime} \in X$ uniquely solves

$$
\int_{0}^{T}\left(a_{r}\left(u^{\prime}(t), v(t)\right)-\left\langle c \dot{u}^{\prime}(t), \dot{v}(t)\right\rangle_{H}\right) \mathrm{d} t=-\int_{0}^{T} a_{h}(u(t), v(t)) \mathrm{d} t
$$

for all $v \in \mathfrak{C}_{0}^{\infty}([0, T], V)$ with homogeneous initial data $u^{\prime}(0)=\dot{u}^{\prime}(0)=0$. In the above right hand side, $u$ is the solution of (4).

Proof. First we show that (20) has a unique solution yielding the well-definedness of $\partial_{2} F(c, r): L^{\infty}(\Omega) \rightarrow X$. To this end we reformulate the right hand side of (20) to fit formulation (9). To each $t \in[0, T]$ we therefore determine the auxiliary function $g(t) \in V$ as unique solution of the elliptic problem

$$
a_{r}(g(t), v)=a_{h}(-u(t), v) \text { for all } v \in \mathfrak{C}_{0}^{\infty}([0, T], V) .
$$

The smoothness of $u$ (Lemma 2.4) transfers to $g$; that is, $g, \dot{g} \in X$. Further,

$$
\|g(t)\|_{V} \lesssim\|h\|_{\infty}\|u(t)\|_{V} \quad \text { and } \quad\|\dot{g}(t)\|_{V} \lesssim\|h\|_{\infty}\|\dot{u}(t)\|_{V} .
$$

In view of Theorem 2.3 problem (20) admits a unique solution with

$$
\left\|u^{\prime}\right\|_{X} \lesssim\|g\|_{\mathrm{e}^{1}([0, T], V)} \lesssim\|h\|_{\infty}\|u\|_{\mathrm{e}^{1}([0, T], V)}
$$

which settles boundedness of $\partial_{2} F$.

Next we validate $\partial_{2} F(c, r)$ as Fréchet derivate of $F$ with respect to $r>\mathrm{k}_{-}$. Let $h \in L^{\infty}$ be so that $r+h \geq \mathrm{k}_{-}$a.e. and denote by $u^{+}$the solution of (4) where $r$ is replaced by $r+h$. The data $u_{0}, u_{1}$, and $f$ are such that $u^{+}$and $u$ have the same regularity (Lemma 2.4). Then, $d:=u^{+}-u-u^{\prime} \in X$ satisfies $d(0)=\dot{d}(0)=0$ as well as

$$
\int_{0}^{T}\left(a_{r}(d(t), v(t))-\langle c \dot{d}(t), \dot{v}(t)\rangle_{H}\right) \mathrm{d} t=\int_{0}^{T} a_{h}\left(u(t)-u^{+}(t), v(t)\right) \mathrm{d} t
$$

for all $v \in \mathcal{C}_{0}^{\infty}([0, T], V)$. Our arguments leading to (21) yield

$$
\|d\|_{X} \lesssim\|h\|_{\infty}\|\delta\|_{\mathrm{e}^{1}([0, T], V)}
$$

where $\delta(t)=u^{+}(t)-u(t)$ satisfies $\delta(0)=\dot{\delta}(0)=0$ as well as

$$
\int_{0}^{T}\left(a_{r}(\delta(t), v(t))-\langle c \dot{\delta}(t), \dot{v}(t)\rangle_{H}\right) \mathrm{d} t=-\int_{0}^{T} a_{h}\left(u^{+}(t), v(t)\right) \mathrm{d} t
$$

for all $v \in \mathcal{C}_{0}^{\infty}([0, T], V)$. Again, arguing as above leads to

$$
\|\delta(t)\|_{V} \lesssim\|h\|_{\infty}\left\|u^{+}\right\|_{\mathcal{C}^{1}([0, T], V)}
$$

where $\left\|u^{+}\right\|_{\mathcal{C}^{1}([0, T], V)}$ stays bounded as $h \rightarrow 0$. Alltogether, $\|d\|_{X} \lesssim\|h\|_{\infty}^{2}$ and we are done.

As in the previous section additional regularity of $r$ allows to weaken the assumptions on the data. 
Corollary 3.6. Let $u_{0} \in H^{2}(\Omega) \cap V, u_{1} \in V$, and $f \in \mathcal{C}^{1}([0, T], H)$. Define

$$
\widetilde{F}: \mathrm{D}(\widetilde{F}) \subset L^{\infty}(\Omega) \times W^{1, \infty}(\Omega) \rightarrow L^{2}([0, T], V), \quad(c, r) \mapsto u,
$$

where $u$ solves (4) and

$$
\mathrm{D}(\widetilde{F})=\left\{(\lambda, \kappa) \in L^{\infty}(\Omega) \times W^{1, \infty}(\Omega): \lambda(\mathbf{x}) \geq \mathrm{k}_{-}, \kappa(\mathbf{x}) \geq \mathrm{k}_{-} \text {a.e. }\right\} .
$$

Then, $\widetilde{F}$ is Fréchet differentiable with respect to $r$ and $\partial_{2} \widetilde{F}(c, r) \in \mathcal{L}\left(W^{1, \infty}(\Omega), X\right)$ where $\partial_{2} \widetilde{F}(c, r) h=u^{\prime}$ with $u^{\prime}$ from (20).

Proof. The data are such that the hypotheses of Lemma 2.4 are met independently of $h \in W^{1, \infty}(\Omega)$. Indeed, $A_{r+h} u_{0} \in H$ for all $h$ with $r+h \geq \mathrm{k}_{-}$. Thus, $u=\widetilde{F}(c, r)$ and $u^{+}=\widetilde{F}(c, r+h)$ have the required regularity to carry over the proof of Theorem 3.5 to the present setting.

\section{FrÉchet Differentiability of the FUlL OPERATOR}

The operator $\mathcal{F}$ mentioned in the Introduction will be defined exactly with the help of the auxiliary map $F$ from (17). Then, the differentiability of $\mathcal{F}$ follows immediately from the differentiability of $F$.

Let

$$
\mathcal{F}: \mathrm{D}(\mathcal{F}) \subset L^{\infty}(\Omega)^{2} \rightarrow X, \quad(\nu, \varrho) \mapsto F\left(\varrho^{-1} \nu^{-2}, \varrho^{-1}\right),
$$

where the domain of definition of $\mathcal{F}$ is

$$
\mathrm{D}(\mathcal{F})=\left\{\lambda \in L^{\infty}(\Omega): 1_{-} \leq \lambda(\mathbf{x}) \leq 1_{+} \text {a.e. }\right\}^{2}
$$

with positive constants $l_{-}<1_{+}$.

The mapping $G: \mathrm{D}(\mathcal{F}) \subset L^{\infty}(\Omega)^{2} \rightarrow L^{\infty}(\Omega)^{2},\left(\begin{array}{c}\nu \\ \varrho\end{array}\right) \mapsto\left(\begin{array}{c}\varrho^{-1} \nu^{-2} \\ \varrho^{-1}\end{array}\right)$, is Fréchet differentiable with

$$
G^{\prime}(\nu, \varrho)\left(\begin{array}{l}
h_{1} \\
h_{2}
\end{array}\right)=-\left(\begin{array}{cc}
2 \varrho^{-1} \nu^{-3} & \varrho^{-2} \nu^{-2} \\
0 & \varrho^{-2}
\end{array}\right)\left(\begin{array}{l}
h_{1} \\
h_{2}
\end{array}\right)=\left(\begin{array}{c}
2 \varrho^{-1} \nu^{-3} h_{1}+\varrho^{-2} \nu^{-2} h_{2} \\
\varrho^{-2} h_{2}
\end{array}\right) .
$$

Further, $G(\mathrm{D}(\mathcal{F})) \subset \mathrm{D}(F)$ whenever $\mathrm{k}_{-} \max \left\{1_{+}, 1_{+}^{3}\right\} \leq 1$.

The chain rule applied to $\mathcal{F}=F \circ G$ yields

$$
\begin{aligned}
\mathcal{F}^{\prime}(\nu, \varrho)\left(\begin{array}{l}
h_{1} \\
h_{2}
\end{array}\right) & =F^{\prime}(G(\nu, \varrho)) G^{\prime}(\nu, \varrho)\left(\begin{array}{l}
h_{1} \\
h_{2}
\end{array}\right) \\
& =-\partial_{1} F(G(\nu, \varrho))\left(2 \varrho^{-1} \nu^{-3} h_{1}+\varrho^{-2} \nu^{-2} h_{2}\right)-\partial_{2} F(G(\nu, \varrho))\left(\varrho^{-2} h_{2}\right)
\end{aligned}
$$

which immediately proves the following theorem.

Theorem 4.1. Let $u_{0}=u_{1}=0$ and $f \in \mathcal{C}^{2}([0, T], H)$ with $f(0)=0$. Then, the Fréchet derivative of $\mathcal{F}$ about $(\nu, \varrho) \in \operatorname{int}(\mathrm{D}(\mathcal{F}))$ exists and is the bounded linear operator $\mathcal{F}^{\prime}(\nu, \varrho) \in \mathcal{L}\left(L^{\infty}(\Omega)^{2}, X\right)$ defined by $\mathcal{F}^{\prime}(\nu, \varrho)\left(h_{1}, h_{2}\right)^{\top}=u^{\prime}$ where $u^{\prime} \in X$ uniquely solves

$$
\begin{aligned}
\int_{0}^{T}\left(a _ { \varrho ^ { - 1 } } \left(u^{\prime}(t)\right.\right. & \left., v(t))-\left\langle\varrho^{-1} \nu^{-2} \dot{u}^{\prime}(t), \dot{v}(t)\right\rangle_{H}\right) \mathrm{d} t \\
& =\int_{0}^{T}\left(a_{h_{2} \varrho^{-2}}(u(t), v(t))-\left\langle\left(2 \varrho^{-1} \nu^{-3} h_{1}+\varrho^{-2} \nu^{-2} h_{2}\right) \dot{u}(t), \dot{v}(t)\right\rangle_{H}\right) \mathrm{d} t
\end{aligned}
$$

for all $v \in \mathfrak{C}_{0}^{\infty}([0, T], V)$ with initial data $u^{\prime}(0)=\dot{u}^{\prime}(0)=0$. In the above right hand side, $u$ is the weak solution of the wave equation (1) with Cauchy data (2), that is, $u=\mathcal{F}(\nu, \varrho)$. 
Moreover, $\mathcal{F}^{\prime}(\nu, \varrho): L^{\infty}(\Omega)^{2} \rightarrow \mathfrak{C}([0, T], H)$ is compact.

Proof. The hypotheses on the data of Theorem 3.3 and 3.5 are satisfied. It remains only to prove the compactness of $\mathcal{F}^{\prime}(\nu, \varrho)=F^{\prime}(G(\nu, \varrho)) G^{\prime}(\nu, \varrho)$. As the Fréchet derivative of a competely continuous mapping is a compact operator, see, e.g. [14, Proposition 7.33], the assertion follows by Theorem 3.2.

The formulation of (22) as a classical partial differential equation is given in the Introduction.

Remark 4.2. The setting of Theorem 4.1 seems quite natural for seismic exploration. Sound speed and mass density cannot be modelled to be smoother than $L^{\infty}(\Omega)$. Further, before firing the energy source $f$ we can reliably assume the environment to be at rest: $u_{0}=u_{1}=f(0)=0$.

In the above remark we justified the strong assumptions on the data $u_{0}, u_{1}$ and $f$. Nevertheless, if we allow smoother $\nu$ and $\rho$ we can weaken the assumptions on $u_{1}$ and $f$ a little bit.

Corollary 4.3. Let $u_{0}=0, u_{1} \in V \cap H^{2}(\Omega)$, and $f \in \mathfrak{C}^{2}([0, T], H)$ with $f(0) \in V$. Define

$$
\widetilde{\mathcal{F}}: \mathrm{D}(\widetilde{\mathcal{F}}) \subset W^{1, \infty}(\Omega)^{2} \rightarrow X, \quad(\nu, \varrho) \mapsto F\left(\varrho^{-1} \nu^{-2}, \varrho^{-1}\right),
$$

where

$$
\mathrm{D}(\widetilde{\mathcal{F}})=\left\{\lambda \in W^{1, \infty}(\Omega): 1_{-} \leq \lambda(\mathbf{x}) \leq 1_{+} \text {a.e. }\right\}^{2} .
$$

Then, $\widetilde{\mathcal{F}}$ is Fréchet differentiable and $\widetilde{\mathcal{F}}^{\prime}(\nu, \varrho) \in \mathcal{L}\left(W^{1, \infty}(\Omega)^{2}, X\right)$ where $\widetilde{\mathcal{F}}^{\prime}(\nu, \varrho)\left(h_{1}, h_{2}\right)=u^{\prime}$ with $u^{\prime}$ from $(22)$.

Remark 4.4. In this remark we discuss the implication of the compactness of $\mathcal{F}^{\prime}(\nu, \varrho)$ : $L^{\infty}(\Omega)^{2} \rightarrow \mathfrak{C}([0, T], H)$ on solving the inverse problem of seismology.

Let $M \subset \Omega$ be the (smooth) measurement submanifold and let the measurement process be modeled by a bounded linear operator $\Psi: \mathcal{C}([0, T], H) \rightarrow L^{2}([0, T] \times M)$. For instance, $\Psi$ could be the trace map $\left.u \mapsto u\right|_{M}$. In this setting the seismic inverse problem reads:

Given measurements $u^{\delta} \in L^{2}([0, T] \times M)$ find $(\nu, \varrho) \in \mathrm{D}(\mathcal{F})$ such that

$$
\Psi \mathcal{F}(\nu, \varrho) \approx u^{\delta} \text {. }
$$

Typical methods to solve above problem are Newton-like iterations which involve repeated approximate solution of local linearizations to obtain the Newton update. Due to the compactness of $\Psi \mathcal{F}^{\prime}(\nu, \varrho): L^{\infty}(\Omega)^{2} \rightarrow L^{2}([0, T] \times M)$ these linear systems are ill-posed. Indeed, compactness yields that the range of $\Psi \mathcal{F}^{\prime}(\nu, \varrho)$ is non-closed which, in turn, yields the stated ill-posedness, see [9]. As a consequence the computation of the Newton update needs to be regularized adequately. Regularization of ill-posed problems in a Banach space setting is a highly topical research issue. For an state of the art overview we refer to the monograph [10] and to the special section Tackling inverse problems in a Banach space environment (Inverse Problems, 28(10), 2012). As $L^{\infty}(\Omega)$ is not reflexive most of the known theory does, unfortunately, not directly apply to our situation. One pragmatic remedy is described in [8, Section 5] but more mathematical research is urgently needed.

Remark 4.5. The Born series is known to converge for the time-harmonic wave equation (Helmholtz equation) provided the frequency is sufficiently small, see, e.g., [5, Chap. 5.2]. Unfortunately, Theorem 4.1 does not provide a rigorous justification of the Born series in time domain for the following reason. We consider the Born series only with respect 
to c. Let $r \in L^{\infty}(\Omega)$ and $f \in \mathrm{C}^{1}([0, T], H)$ be fixed and $u, u^{+} \in X$ be the solutions of (1), (2) for $c$ and $c+h$, respectively. The difference $\delta=u^{+}-u$ solves $\delta(0)=\dot{\delta}(0)=0$ and

$$
\begin{aligned}
\int_{0}^{T}\left(a_{r}(\delta(t), v(t))-\langle c \dot{\delta}(t), \dot{v}(t)\rangle_{H}\right) \mathrm{d} t & =\int_{0}^{T}\left\langle h \dot{u}^{+}(t), \dot{v}(t)\right\rangle_{H} \mathrm{~d} t \\
& =-\int_{0}^{T}\left\langle h \ddot{u}^{+}(t), v(t)\right\rangle_{H} \mathrm{~d} t
\end{aligned}
$$

for all $v \in \mathcal{C}_{0}^{\infty}([0, T], V)$. Define the operator $\mathcal{L}: L^{2}([0, T], H) \rightarrow X$ by $f \mapsto u$ where $u \in X$ solves (1) for $c$ and $r$ with $u(0)=\dot{u}(0)=0$. Then the previous variational equation can be written as $u^{+}-u=-\mathcal{L}\left(h \ddot{u}^{+}\right)$. This is a fixed point equation for $u^{+}$. The first step in the iteration procedure yields the Born approximation, that is, $u_{B}=u-\mathcal{L}(h \ddot{u})$. Convergence of the iteration sequence in some space $Y$ is proven if the norm of the linear operator $v \mapsto \mathcal{L}(h \ddot{v})$ from $Y$ into itself is less than one. However, this operator does not seem to be bounded - or even well defined - in any reasonable norm because $\mathcal{L}$ does not compensate for the second derivative. The fundamental reason for this shortcoming is the fact that (4) does not admit a solution $u \in L^{2}([0, T], V)$ for $f \in L^{2}\left([0, T], V^{\prime}\right)$ as we have demonstrated in Example 2.1.

\section{Comparison to the work of Blazek, Stolk, and Symes}

Blazek, Stolk, and Symes [3] considered inverse wave problems in the framework of hyperbolic systems of integro-differential equations with bounded and measurable coefficients. Among other things they showed Fréchet differentiability with respect to the coefficients. In this section we relate their results to ours.

To this end we formulate a first order system leading to (1). Let $p=p(\mathbf{x}, t) \in \mathbb{R}$ and $\mathbf{v}=\mathbf{v}(\mathbf{x}, t) \in \mathbb{R}^{d}$ be functions defined on $\Omega \times \mathbb{R}$ where $\Omega \subset \mathbb{R}^{d}$ is a domain. Let $p$ and $\mathbf{v}$ satisfy $p(\cdot, 0)=0, \mathbf{v}(\cdot, 0)=\mathbf{0},\left.p\right|_{\partial \Omega}=0$, and

$$
\begin{aligned}
& c \partial_{t} p=-\nabla_{\mathbf{x}} \cdot \mathbf{v}+f, \\
& b \partial_{t} \mathbf{v}=-\nabla_{\mathbf{x}} p+\mathbf{g},
\end{aligned}
$$

in $\Omega \times[0, \infty[$. Further, $\mathbf{g}=\mathbf{g}(\mathbf{x}, t)$ and $f=f(\mathbf{x}, t)$ are external forces. The bounded inhomogeneities $c=c(\mathbf{x})$ and $b=b(\mathbf{x})$ are also bounded away from zero.

Define $\mathbf{G}(\cdot, t)=\int_{0}^{t} \mathbf{g}(\cdot, s) \mathrm{d} s$ and assume the scalar-valued function $w=w(\mathbf{x}, t)$ solves the wave equation

$$
c \partial_{t}^{2} w-\nabla_{\mathbf{x}} \cdot\left(\frac{1}{b} \nabla_{\mathbf{x}} w\right)=f-\nabla_{\mathbf{x}} \cdot\left(\frac{1}{b} \mathbf{G}\right),\left.\quad w\right|_{\partial \Omega}=0
$$

with $w(\cdot, 0)=\partial_{t} w(\cdot, 0)=0$. Then, $p=\partial_{t} w$ and $\mathbf{v}=-\frac{1}{b}\left(\nabla_{\mathbf{x}} w-\mathbf{G}\right)$ solve the above first order system, and vice versa.

Blazek, Stolk, and Symes [3] studied abstract evolution problems like the following: Let $U$ be a real separable Hilbert space. Find an $U$-valued function $\xi=\xi(t)$ which solves (in a suitable sense)

$$
A \dot{\xi}+P \xi=\ell, \quad \xi(0)=0,
$$

in which the right hand side $\ell=\ell(t)$ is also $U$-valued. Further, $P$ is a skew-symmetric operator with domain $V$ being a dense subspace of $U$ with a topology that is stronger than the one induced by $U$. The operator $A \in \mathcal{L}(U)$ is self-adjoint and coercive. 
System (23) can be written in the abstract form when setting $U=L^{2}(\Omega)^{1+d}, V=$ $H_{0}^{1}(\Omega) \times H_{\mathrm{div}}^{1}(\Omega)$,

$$
\xi=\left(\begin{array}{l}
p \\
\mathbf{v}
\end{array}\right), A(c, b)=\left(\begin{array}{cccc}
c & 0 & 0 & 0 \\
0 & b & 0 & 0 \\
0 & 0 & b & 0 \\
0 & 0 & 0 & b
\end{array}\right), P=\left(\begin{array}{cccc}
0 & \partial_{x_{1}} & \partial_{x_{2}} & \partial_{x_{3}} \\
\partial_{x_{1}} & 0 & 0 & 0 \\
\partial_{x_{2}} & 0 & 0 & 0 \\
\partial_{x_{3}} & 0 & 0 & 0
\end{array}\right), \text { and } \ell=\left(\begin{array}{c}
f \\
\mathbf{g}
\end{array}\right)
$$

see $[3$, Appendix A] for the skew-symmetry of $P$.

We now paraphrase Theorem 9 of [3] concerning the Fréchet-differentiability of the map $\mathcal{S}: \mathrm{D}(\mathcal{S}) \subset \mathcal{L}_{\text {sym }}(U) \rightarrow \mathcal{C}^{0}([0, T], V), A \mapsto \xi$, where $\mathcal{L}_{\text {sym }}(U)$ is the Banach space of self-adjoint operators acting on $U$ and $\mathrm{D}(\mathcal{S})=\left\{B \in \mathcal{L}_{\mathrm{sym}}(U): \beta_{1} I \leq B \leq \beta_{2} I\right\}$ with constants $0<\beta_{1} \leq \beta_{2}$.

If $\ell \in H_{\text {loc }}^{2}(\mathbb{R}, U), \operatorname{supp} \ell \subset[0, \infty[$, then $\mathcal{S}$ is (continuously) Fréchet-differentiable with $\mathcal{S}^{\prime}(A) H=\xi^{\prime}$ and $\xi^{\prime}$ is the unique solution of

$$
A \dot{\xi}^{\prime}+P \xi^{\prime}=-H \dot{\xi}, \quad \xi^{\prime}(0)=0,
$$

where $\xi$ uniquely solves (25).

We apply this result to (23) via (26). Let $S: \mathrm{D}(S) \subset L^{\infty}(\Omega)^{2} \rightarrow \mathrm{C}^{0}\left([0, T], H_{0}^{1}(\Omega) \times\right.$ $\left.H_{\text {div }}^{1}(\Omega)\right),(c, b) \mapsto(p, \mathbf{v})$, where $\mathrm{D}(S)=\left\{q \in L^{\infty}(\Omega): \beta_{1} \leq q(\mathbf{x}) \leq \beta_{2}\right\}^{2}$. Since $S(c, b)=$ $\mathcal{S}(A(c, b))$ we have that

$$
S^{\prime}(c, b)\left(h_{1}, h_{2}\right)=\mathcal{S}^{\prime}(A(c, b)) A^{\prime}(c, b)\left(h_{1}, h_{2}\right)=\mathcal{S}^{\prime}(A(c, b)) H
$$

where $H=\operatorname{diag}\left(h_{1}, h_{2}, h_{2}, h_{2}\right)$. In other words: $S^{\prime}(c, b)\left(h_{1}, h_{2}\right)=\left(p^{\prime}, \mathbf{v}^{\prime}\right)$ where $p^{\prime}(\cdot, 0)=$ $0, \mathbf{v}^{\prime}(\cdot, 0)=\mathbf{0},\left.p^{\prime}\right|_{\partial \Omega}=0$, and

$$
\begin{aligned}
c \partial_{t} p^{\prime} & =-\nabla_{\mathbf{x}} \cdot \mathbf{v}^{\prime}-h_{1} \partial_{t} p, \\
b \partial_{t} \mathbf{v}^{\prime} & =-\nabla_{\mathbf{x}} p^{\prime}-h_{2} \partial_{t} \mathbf{v},
\end{aligned}
$$

which is equivalent to $w^{\prime}(\cdot, 0)=\partial_{t} w^{\prime}(\cdot, 0)=0,\left.w^{\prime}\right|_{\partial \Omega}=0$,

$$
\begin{aligned}
c \partial_{t}^{2} w^{\prime}-\nabla_{\mathbf{x}} \cdot\left(\frac{1}{b} \nabla_{\mathbf{x}} w^{\prime}\right) & =-h_{1} \partial_{t} p+\nabla_{\mathbf{x}} \cdot\left(\frac{h_{2}}{b} \mathbf{v}\right) \\
& =-h_{1} \partial_{t}^{2} w-\nabla_{\mathbf{x}} \cdot\left(\frac{h_{2}}{b^{2}} \nabla_{\mathbf{x}} w\right)+\nabla_{\mathbf{x}} \cdot\left(\frac{h_{2}}{b^{2}} \mathbf{G}\right)
\end{aligned}
$$

by $p^{\prime}=\partial_{t} w^{\prime}$ and $\mathbf{v}^{\prime}=-\frac{1}{b}\left(\nabla_{\mathbf{x}} w^{\prime}+h_{2} \mathbf{v}\right)$.

Finally, let $\Phi: \mathrm{D}(S) \subset L^{\infty}(\Omega)^{2} \rightarrow \mathrm{C}^{0}\left([0, T], H_{0}^{1}(\Omega)\right)$ be defined by $\Phi(c, b)=w(\cdot, t)=$ $\int_{0}^{t} p(\cdot, s) \mathrm{d} s$ where $p$ and $w$ solve $(23)$ and $(24)$, respectively, with $\mathbf{G}=\mathbf{g}=\mathbf{0}$ and $f \in$ $H_{\text {loc }}^{2}\left(\mathbb{R}, L^{2}(\Omega)\right), \operatorname{supp} f \subset\left[0, \infty\left[\right.\right.$. As $p=S_{1}(c, b)$ (first component of $S$ ) we obtain

$$
\Phi^{\prime}(c, b)\left(h_{1}, h_{2}\right)=\int_{0}^{t} p^{\prime}(\cdot, s) \mathrm{d} s=w^{\prime}(\cdot, t)
$$

where $w^{\prime}$ and $p^{\prime}$ are the solutions of $(28)$ and $(27)$, respectively $(\mathbf{G}=\mathbf{0})$.

We see that (29) would also have been obtained by our findings from the previous sections (Theorems 3.3 and 3.5). Indeed, let $F$ denote the operator defined in (17). Then, $\Phi(c, b)=F(c, 1 / b)$ and

$$
\Phi^{\prime}(c, b)\left(h_{1}, h_{2}\right)=F^{\prime}\left(c, \frac{1}{b}\right)\left(h_{1},-\frac{h_{2}}{b^{2}}\right) .
$$


Observe, that our assumption on $f$ is a little bit less restrictive: $f \in H_{\text {loc }}^{2}\left(\mathbb{R}, L^{2}(\Omega)\right)$, $\operatorname{supp} f \subset[0, T]$ (Blazek et al.) vs. $f \in \mathrm{C}^{2}\left([0, T], L^{2}(\Omega)\right), f(0)=0$. In view of Remark 4.2 this difference does not matter though for seismic applications.

\section{REFERENCES}

[1] G. BAO, Smoothness between coefficients and boundary values for the wave equation, SIAM J. Math. Anal., 29 (1998), pp. 380-394.

[2] G. Bao And W. W. Symes, On the sensitivity of solutions of hyperbolic equations to the coefficients, Comm. Partial Differential Equations, 21 (1996), pp. 395-422.

[3] K. D. Blazek, C. Stolk, and W. W. Symes, A mathematical framework for inverse wave problems in heterogeneous media, Inverse Problems, 29 (2013), p. 065001.

[4] L. C. Evans, Partial differential equations, vol. 19 of Graduate Studies in Mathematics, American Mathematical Society, Providence, RI, second ed., 2010.

[5] A. KIRsch, An introduction to the mathematical theory of inverse problems, vol. 120 of Applied Mathematical Sciences, Springer, New York, second ed., 2011.

[6] S. LANG, Real and functional analysis, vol. 142 of Graduate Texts in Mathematics, Springer-Verlag, New York, third ed., 1993.

[7] J. L. Lions And E. Magenes, Non-Homogeneous Boundary Value Problems and Applications, Vol. 1, Springer-Verlag, New York, 1972.

[8] F. Margotti, A. Rieder, And A. Leitão, A Kaczmarz version of the REGINN-Landweber iteration for ill-posed problems in Banach spaces, Tech. Rep. 13/02, Institute for Scientific Computing and Mathematical Modelling, KIT, Germany, 2013. Submitted. Available online from http: //www.math.kit.edu/iwrmm/seite/preprints/media/preprint\%20nr.\%2013-02.pdf.

[9] M. Z. NAshed, A new approach to classification and regularization of ill-posed operator equations, in Inverse and Ill-Posed Problems, H. W. Engl and C. W. Groetsch, eds., vol. 4 of Notes and Reports in Mathematics in Science and Engineering, Boston, 1987, Academic Press, pp. 53-75.

[10] T. Schuster, B. Kaltenbacher, B. Hofmann, and K. S. Kazimierski, Regularization methods in Banach spaces, vol. 10 of Radon Series on Computational and Applied Mathematics, Walter de Gruyter GmbH \& Co. KG, Berlin, 2012.

[11] C. Stolk, On the modeling and inversion of seismic data, PhD thesis, University of Utrecht, The Netherlands, 2000. http://www.library.uu.nl/digiarchief/dip/diss/1944076/full.pdf.

[12] W. W. Symes, The seismic reflection inverse problem, Inverse Problems, 25 (2009), pp. 123008, 39.

[13] J. Wloka, Partial Differential Equations, Cambridge University Press, Cambridge, U. K., 1987.

[14] E. ZEIDLER, Nonlinear Functional Analysis and its Applications I: Fixed-Point Theorems, SpringerVerlag, New York, 1993.

Department of Mathematics, Karlsruhe Institute of Technology (KIT), D-76128 KarlsRUhe, Germany

E-mail address: andreas.kirsch@kit.edu

E-mail address: andreas.rieder@kit.edu 


\section{IWRMM-Preprints seit 2011}

Nr. 11/01 Tobias Jahnke, Derya Altintan : Efficient simulation of discret stochastic reaction systems with a splitting method

Nr. 11/02 Tobias Jahnke : On Reduced Models for the Chemical Master Equation

Nr. 11/03 Martin Sauter, Christian Wieners : On the superconvergence in computational elastoplasticity

Nr. 11/04 B.D. Reddy, Christian Wieners, Barbara Wohlmuth : Finite Element Analysis and Algorithms for Single-Crystal Strain-Gradient Plasticity

Nr. 11/05 Markus Bürg: An hp-Efficient Residual-Based A Posteriori Error Estimator for Maxwell's Equations

Nr. 12/01 Branimir Anic, Christopher A. Beattie, Serkan Gugercin, Athanasios C. Antoulas: Interpolatory Weighted-H2 Model Reduction

Nr. 12/02 Christian Wieners, Jiping Xin: Boundary Element Approximation for Maxwell's Eigenvalue Problem

Nr. 12/03 Thomas Schuster, Andreas Rieder, Frank Schöpfer: The Approximate Inverse in Action IV: Semi-Discrete Equations in a Banach Space Setting

Nr. 12/04 Markus Bürg: Convergence of an hp-Adaptive Finite Element Strategy for Maxwell's Equations

Nr. 12/05 David Cohen, Stig Larsson, Magdalena Sigg: A Trigonometric Method for the Linear Stochastic Wave Equation

Nr. 12/06 Tim Kreutzmann, Andreas Rieder: Geometric Reconstruction in Bioluminescence Tomography

Nr. 12/07 Tobias Jahnke, Michael Kreim: Error bound for piecewise deterministic processes modeling stochastic reaction systems

Nr. 12/08 Haojun Li, Kirankumar Hiremath, Andreas Rieder, Wolfgang Freude: Adaptive Wavelet Collocation Method for Simulation of Time Dependent Maxwell's Equations

Nr. 12/09 Andreas Arnold, Tobias Jahnke: On the approximation of high-dimensional differential equations in the hierarchical Tucker format

Nr. 12/10 Mike A. Botchev, Volker Grimm, Marlis Hochbruck: Residual, Restarting and Richardson Iteration for the Matrix Exponential

Nr. 13/01 Willy Dörfler, Stefan Findeisen: Numerical Optimization of a Waveguide Transition Using Finite Element Beam Propagation

Nr. 13/02 Fabio Margotti, Andreas Rieder, Antonio Leitao: A Kaczmarz Version of the ReginnLandweber Iteration for Ill-Posed Problems in Banach Spaces

Nr. 13/03 Andreas Kirsch, Andreas Rieder: On the Linearization of Operators Related to the Full Waveform Inversion in Seismology

Eine aktuelle Liste aller IWRMM-Preprints finden Sie auf: 


\section{Kontakt}

Karlsruher Institut für Technologie (KIT) Institut für Wissenschaftliches Rechnen und Mathematische Modellbildung

Prof. Dr. Christian Wieners Geschäftsführender Direktor

Campus Süd

Engesserstr. 6

76131 Karlsruhe

E-Mail:Bettina.Haindl@kit.edu

www. math. kit.edu/iwrmm/

\section{Herausgeber}

Karlsruher Institut für Technologie (KIT) Kaiserstraße 12 | 76131 Karlsruhe

Juni 2013 